\title{
Incretin-based therapies for patients with type 1 diabetes: a meta-analysis
}

\author{
Lili Liu' ${ }^{1,2}$, Zhuo Shao ${ }^{3}$, Ying Xia ${ }^{1,2}$, Jiabi Qin ${ }^{4}$, Yang Xiao ${ }^{1,2}$, Zhiguang Zhou ${ }^{1,2}$ and Zubing Mei ${ }^{5}$ \\ ${ }^{1}$ Department of Metabolism \& Endocrinology, The Second Xiangya Hospital, Central South University, Changsha, Hunan, China \\ ${ }^{2}$ Key Laboratory of Diabetes Immunology, Central South University, Ministry of Education, National Clinical Research Center for Metabolic Diseases, \\ Changsha, Hunan, China \\ ${ }^{3}$ Department of General Surgery, Changhai Hospital, The Second Military Medical University, Shanghai, Shanghai, China \\ ${ }^{4}$ Department of Epidemiology \& Health Statistics, Xiangya School of Public Health, Central South University, Changsha, Hunan, China \\ ${ }^{5}$ Department of Anorectal Surgery, Shuguang Hospital, Shanghai University of Traditional Chinese Medicine, Shanghai, China
}

Correspondence should be addressed to Y Xiao or Z Zhou: xiaoyang29@csu.edu.cn or zhouzhiguang@csu.edu.cn

\begin{abstract}
Objective: Combined treatment with an incretin-based drug, such as a glucagon-like peptide 1 receptor agonist (GLP-1 RA) or a dipeptidyl peptidase-4 (DPP-4) inhibitor, and basal insulin is a new strategy for improving glucose control in type 1 diabetes mellitus (T1DM). We performed a meta-analysis to assess the effect of this combined treatment on glycaemic control, insulin dose, severe hypoglycaemia, weight gain and gastrointestinal side effects in T1DM patients.

Methods: We searched PubMed, EMBASE and the Cochrane Library for relevant studies published before July 16,2018 . The primary outcome was glycosylated haemoglobin (HbA1c). Secondary outcomes included total daily insulin dose, body weight, severe hypoglycaemia and gastrointestinal side effects.

Results: Nine randomized controlled trials (RCTs) involving 2389 patients were ultimately included in the meta-analysis. The pooled data suggested that incretin-based therapy was associated with a reduction in $\mathrm{HbA} 1 \mathrm{c}$ levels (weighted mean difference (WMD) $-0.17 \%, 95 \%$ confidence interval $(\mathrm{Cl})-0.24$ to $-0.11, P<0.001)$, total daily insulin dose (WMD $-5.53 \mathrm{IU} /$ day, $95 \% \mathrm{Cl}-8.89$ to $-2.17, P=0.001$ ) and body weight (WMD $-3.24 \mathrm{~kg}, 95 \% \mathrm{Cl}-4.43$ to $-2.04, P<0.001)$. Incretins did not increase the risk of severe hypoglycaemia (odds ratio (OR) $0.83,95 \% \mathrm{Cl} 0.60-1.16, P=0.287$ ) but increased the occurrence of gastrointestinal side effects (OR 3.46, 95\% CI 2.20-5.45, $P<0.001$ ). Conclusions: In T1DM patients, GLP-1 RAs, but not DPP-4 inhibitors, combined with insulin appear to be an effective therapy but may increase the occurrence of gastrointestinal side effects.
\end{abstract}

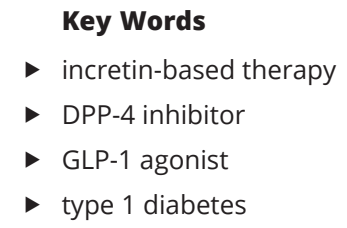

\section{Introduction}

Type 1 diabetes mellitus (T1DM), which is caused by the selective destruction of the insulin-producing $\beta$ cells located in the pancreatic islets of Langerhans, is one of the most common and debilitating autoimmune diseases (1). Usually, treatment with multiple daily injections (MDI) of prandial rapid-acting insulin and basal long-acting insulin or continuous subcutaneous insulin infusion (CSII) is recommended for patients with T1DM to obtain

satisfactory glycaemic control (2). According to the Diabetes Control and Complications Trial Research Group, intensive insulin therapy can effectively delay the onset and slow the progression of chronic diabetic microvascular complications, such as diabetic retinopathy, nephropathy and neuropathy (3). However, adverse events, such as severe hypoglycaemia and weight gain, are also strongly associated with long-term administration of insulin (4). 
To overcome this shortcoming, the development of new therapy agents is urgently needed.

Incretin-based drugs, including glucagon-like peptide 1 receptor agonists (GLP-1 RAs) and dipeptidyl peptidase 4 (DPP-4) inhibitors, may offer an opportunity to avoid these side effects. Theoretically, GLP-1 RAs are structurally and functionally similar to GLP-1 and can mimic the effects of GLP-1, such as augmenting glucose-dependent insulin secretion, suppressing glucagon secretion, delaying gastric emptying $(5,6,7,8)$ and finally enhancing blood glucose management with weight loss (9). DPP-4 inhibitors inhibit the degradation of endogenous GLP-1 and GIP, resulting in reduced glucose. DPP-4 inhibitors are multitarget drugs (10) and can modulate immune function in autoimmune diabetes (11). GLP-1 RAs can provide robust glucosecontrol capability in type 2 diabetes (12). These drugs were both approved by the US Food and Drug Administration (FDA) and have been widely used in patients with type 2 diabetes $(13,14)$ but not in those with type 1 diabetes. Liraglutide and sitagliptin are representative GLP-1 RAs and DPP-4 inhibitors, respectively.

Recently, several studies showed that adding liraglutide to insulin treatment in subjects with type 1 diabetes reduced glycosylated haemoglobin (HbA1c) and body weight, but these benefits were accompanied by an increased risk of hypoglycaemia $(15,16)$. However, some other studies reported opposite results. The Gejgaard group found that liraglutide could not significantly lower HbA1c and body weight, despite the marked reduction in hypoglycaemic events (17). Kuhadiya conducted a study in 2016, and the results showed that different doses of liraglutide exerted different effects on HbA1c and body weight but had no significant difference in per cent time spent in hypoglycaemia (18). Although sitagliptin increased post-meal GLP-1 levels, it did not change HbA1c, body weight or the rate of hypoglycaemia progression (19). Due to the conflicting results, we collected all the available data and conducted a meta-analysis to robustly evaluate the influence of incretin-based therapy added to insulin vs placebo or no drug added to insulin in subjects with type 1 diabetes on the key outcomes HbA1c, total insulin dose, body weight, severe hypoglycaemia and gastrointestinal side effects.

\section{Methods}

\section{Protocol and guidance}

An elaborate protocol of literature-search strategies, inclusion and exclusion criteria, study selection, data extraction, outcome measurements and methods of statistical analysis was prepared with prioritization according to the Preferred Reporting Items for Systematic Reviews and Meta-Analysis (PRISMA) (20).

\section{Literature-search strategy}

Relevant studies published before July 16, 2018 were selected from PubMed, EMBASE and the Cochrane Library. There was no restriction in searching language, publication year or region. Systematically searching for likely eligible studies, we used MeSH terms and their combinations: (type 1 diabetes mellitus) AND (dipeptidyl peptidase 4 or glucagon-like peptide 1). The complete database search strategies are shown in Supplementary data (see section on supplementary data given at the end of this article). In addition, we searched ClinicalTrials.gov to obtain more details and explicit information about the relevant clinical trials.

\section{Inclusion and exclusion criteria}

The inclusion criteria of this meta-analysis were as follows. First, the studies were randomized controlled trials (RCTs). Second, they adopted a parallel design of GLP-1 RAs or DPP-4 inhibitors and insulin vs placebo treatment (or single insulin therapy). Third, the subjects of the studies were restricted to patients with type 1 diabetes, including those with latent autoimmune diabetes in adults (LADA), as LADA is a form of adult-onset autoimmune diabetes or a subtype of T1DM (21). Finally, the studies reported at least one of the following results: HbA1c change (from baseline to endpoint), total insulin dose, weight loss or hypoglycaemia.

The exclusion criteria included (1) editorials, review articles, case reports, letters to the editor and other nonRCTs; (2) duplications of the searched studies and (3) other studies that did not meet the above inclusion criteria.

\section{Data extraction and outcomes of interest}

After skimming through titles/abstracts and full texts, data from the included publications were extracted independently by two of the well-trained authors (Liu L and Xiao Y). These authors also assessed the risk of bias of the eligible studies with detailed instructions. Any disagreements were resolved by discussion or the senior authors (Zhou Z and Xiao Y).

From eligible RCTs, the following information was extracted: authors, publication year, ethnicity, design,

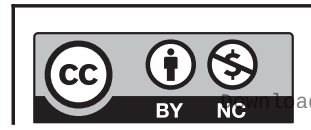

This work is licensed under a Creative Commons Attribution-NonCommercial 4.0 International License. ded from Bioscientifica.com at 04/26/2023 07:39:21AM 
GLP-1 RAs or DPP-4 inhibitor therapy, duration of interventions, sample size, mean age, mean $\mathrm{HbA1c}$, BMI and duration of diabetes. In the case of multiple studies describing the same population, the most recent or complete study was selected.

The primary outcome was HbA1c, and the secondary outcomes were total daily insulin dose, body weight, the occurrence rate of severe hypoglycaemic events, gastrointestinal side effects from the baseline to the end of treatments and hyperglycaemia with ketosis.

\section{Statistical analysis and assessment of study quality and publication bias}

The pooled statistics of the trials were calculated and analysed with Review Manager, version 5.3 (Cochrane Collaboration, Oxford, UK) and Stata/SE 14.0 software (StataCorp). The weighted mean difference (WMD) was adopted to compare continuous variables (HbA1c, total daily insulin dose, body weight), and the odds ratio (OR) was used to compare dichotomous variables (events of severe hypoglycaemia and gastrointestinal side effects). The results were reported with WMD or OR with 95\% confidence intervals (CIs) for continuous and dichotomous variables, respectively. In terms of studies that presented data as the means and range values, the standard deviations (s.D.s) were calculated using the technique described by Wan et al. (22).

To assess the risk of bias of the RCTs, two reviewers independently used a modified Cochrane risk of bias instrument (23). The risks were mainly associated with selection bias, performance bias, detection bias, attrition bias and reporting bias. The items included random sequence generation, allocation concealment, blinding of the participants and researchers, incomplete outcome data, selective reporting and other bias. Due to the small number of included studies, funnel plots and Egger's tests were not performed to screen for potential publication bias (24).

Statistical heterogeneity between studies was evaluated using the chi-square test with significance set at $P \leq 0.10$, and heterogeneity was quantified by the $I^{2}$ statistic with an $I^{2}$ less than 50\% indicating low heterogeneity (25). The fixed-effects model was adopted if there was no significant heterogeneity among studies; otherwise, the randomeffects model was employed.

Due to different study designs, the types of therapy, drug dosages, duration of intervention and different BMI of enrolled participants might have different effects. Subgroup analysis was performed to explore the source of heterogeneity: (1) study design (double-blind RCT vs open-label RCT); (2) types of therapy (GLP-1 RAs or DPP-4 inhibitors); (3) treatment dose of liraglutide (1.8, 1.2 or $0.6 \mathrm{mg}$ ); (4) duration of intervention ( $\leq 12$ weeks or $>12$ weeks) and (5) mean baseline BMI $\left(\geq 28 \mathrm{~kg} / \mathrm{m}^{2}\right.$ or $\left.<28 \mathrm{~kg} / \mathrm{m}^{2}\right)$.

\section{Results}

\section{Search results and characteristics of the included studies}

We initially screened 2398 relevant publications from PubMed, EMBASE and the Cochrane Library. According to the inclusion and exclusion criteria, we finally selected nine RCTs $(15,16,17,18,19,26,27,28,29)$ for the metaanalysis. The detailed process for study selection is shown in Fig. 1. Six $(66.7 \%)(15,16,17,18,28,29)$ RCTs tested GLP-1 RAs (liraglutide dose from $0.6 \mathrm{mg}$ to $1.8 \mathrm{mg}$ ), two $(22.2 \%)(19,27)$ RCTs tested DPP-4 inhibitors and one (11.1\%) (26) RCT investigated both agents.

The characteristics of the nine enrolled studies are shown in Table 1 . The nine trials were all published between 2013 and 2017. Seven (77.8\%) (15, 16, 17, 18, 19, $28,29)$ double-blind RCTs were performed with Caucasians and two $(22.2 \%)(26,27)$ open-label RCTs were performed with Asians. The duration of interventions ranged from 12 weeks to 52 weeks. The total number of individuals enrolled in each of the nine studies ranged from 17 to 1389. In 6 out of 9 studies, the men outnumbered the women, and the male proportion varied from 44 to $72 \%$. The mean age for the included trials was between 28 and 48 years. The mean baseline HbA1c was from 6.5 to $9.7 \%$. The mean baseline weight ranged from 57.3 to $93.7 \mathrm{~kg}$, while the BMI ranged from 21.5 to $30.0 \mathrm{~kg} / \mathrm{m}^{2}$. The mean disease duration of patients in seven clinical trials studying long-standing T1DM ranged from 19.0 to 24.0 years. The average disease durations of patients with newly diagnosed T1DM and LADA were 0.1 and 1.4 years, respectively.

\section{Risk of bias in the enrolled studies}

The evaluated risk of bias for the eligible studies is shown in Fig. 2. All studies used random sequence generation, seven were double-blind trials and the others were open-label trials. Three studies did not mention any information about allocation concealment. Descriptions of the blinding of the outcome assessment were not provided in three trials. One trial had incomplete

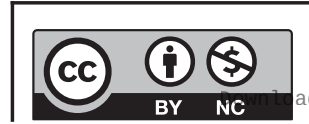

This work is licensed under a Creative Commons Attribution-NonCommercial 4.0 International License. ded from Bioscientifica.com at 04/26/2023 07:39:21AM 


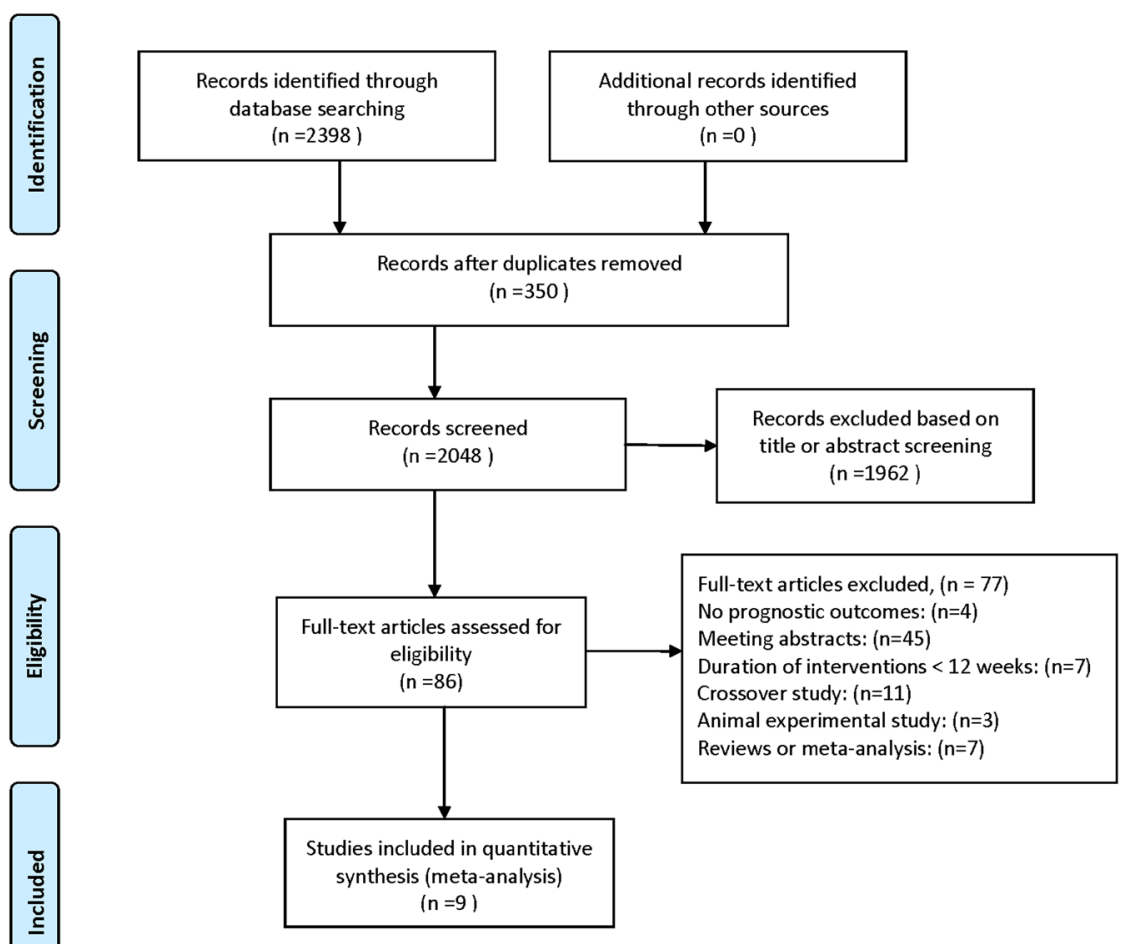

Figure 1

Flow diagram of studies identified, included and excluded.

outcome data, and there was no selective reporting. Other biases were unclear.

\section{Primary outcomes of interest}

\section{$\mathrm{HbA1c}$ reduction in T1DM patients treated with incretin-based therapy and a subgroup analysis of HbA1c based on the study design (double-blind or open-label), drug type (GLP-1 RAs or DPP-4 inhibitors), liraglutide dose $(1.8,1.2$ or $0.6 \mathrm{mg})$, duration of intervention ( $\leq 12$ weeks or $>12$ weeks) and mean baseline BMI ( $\geq 28 \mathrm{~kg} / \mathrm{m}^{2}$ or $<28 \mathrm{~kg} / \mathrm{m}^{2}$ )}

Changes in HbA1c from baseline to endpoint were reported in all nine studies $(15,16,17,18,19,26,27$, 28, 29). As shown in Fig. 3, HbA1c decreased significantly in the incretin-based therapy group compared with the control group (WMD $-0.17 \%, 95 \%$ CI -0.24 to -0.11 ), and the heterogeneity was low $\left(I^{2}=0 \%, P=0.53\right)$.

The nine included studies were categorized as double-blind studies $(n=7)$ and open-label studies $(n=2)$. A significant reduction in HbA1c was observed in the double-blind studies with a large sample size (WMD $-0.18,95 \%$ CI -0.24 to -0.11 ) but not in the open-label studies (WMD 0, 95\% CI -0.42 to 0.42).

Regarding data limited to a single type of incretinbased therapy, a significant reduction in $\mathrm{HbA1c}$ was found in the GLP-1 RA group (WMD $-0.2,95 \%$ CI -0.26 to
-0.13, $P<0.001)$ with low heterogeneity $\left(I^{2}=0 \%, P=0.85\right)$ but not in the DPP-4 inhibitor group (WMD 0.05, 95\% CI -0.17 to $0.27, P=0.67)$ with low heterogeneity $\left(I^{2}=0 \%\right.$, $P=0.92$ ) (Table 2).

Compared with controls, the incretin-based drug liraglutide reduced $\mathrm{HbA1c}$ in a dose-dependent manner. Treatment with $1.8 \mathrm{mg}$ liraglutide (WMD -0.24, 95\% CI -0.32 to $-0.16, P<0.001$ ), $1.2 \mathrm{mg}$ liraglutide (WMD -0.2 , $95 \% \mathrm{CI}-0.28$ to $-0.11, P<0.001)$ and $0.6 \mathrm{mg}$ liraglutide (WMD $-0.15,95 \%$ CI -0.24 to $-0.07, \quad P<0.001$ ) significantly decreased HbA1c levels (test for subgroup difference: $P=0.35$ ). Heterogeneity was low with the chisquare test $I^{2}<50 \%$ and $P>0.1$ for all three subgroups (Table 2).

In exploratory analyses, the subgroup analysis based on the duration of the intervention showed that in the group treated with the intervention for a duration less than 12 weeks, there was no significant difference in the reduction in $\mathrm{HbA1c}$ after the incretin-based treatment (WMD $-0.13,95 \% \mathrm{CI}-0.39$ to $0.13, P=0.31$ ). In the group with the prolonged intervention for more than 12 weeks, HbA1c was significantly improved after the incretin-based treatment (WMD $-0.18,95 \% \mathrm{CI}-0.24$ to $-0.11, P<0.001$ ) (Table 2).

In the subgroup with a mean baseline BMI $\geq 28 \mathrm{~kg} / \mathrm{m}^{2}$, there was a significant decrease in the HbA1c levels after the incretin-based treatment (WMD -0.20,

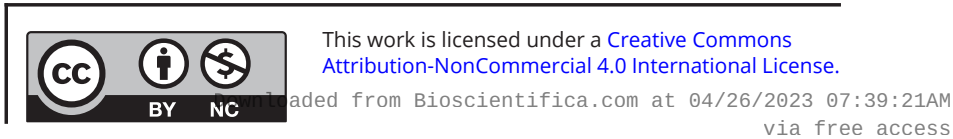




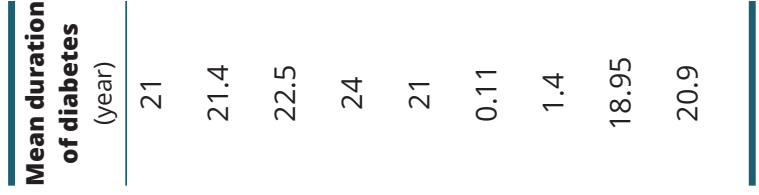

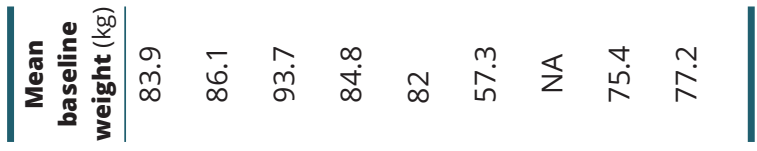

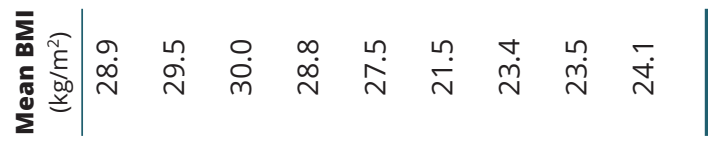

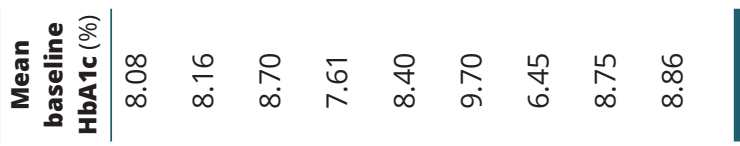

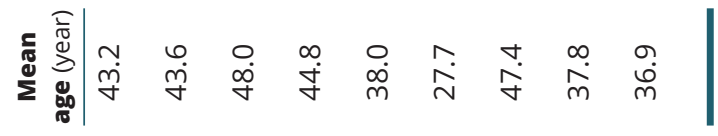

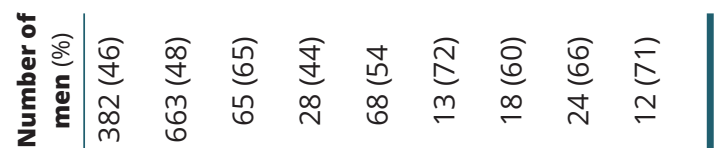

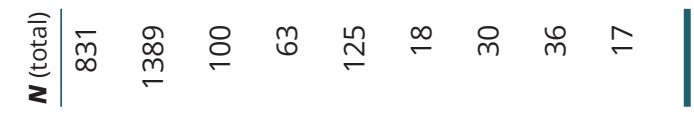

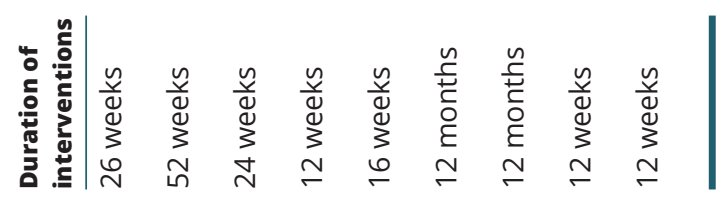

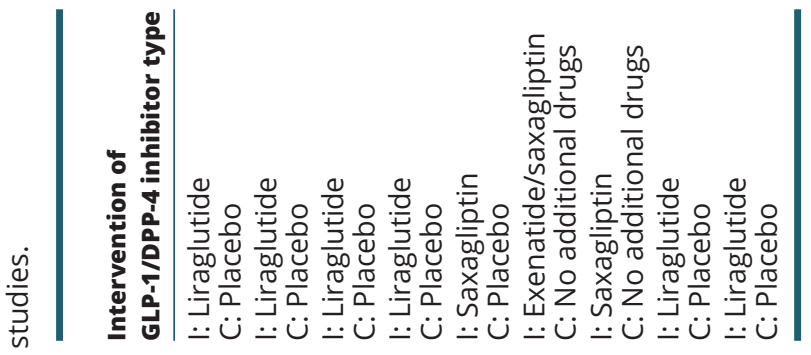



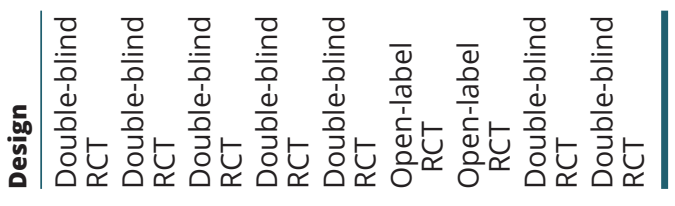

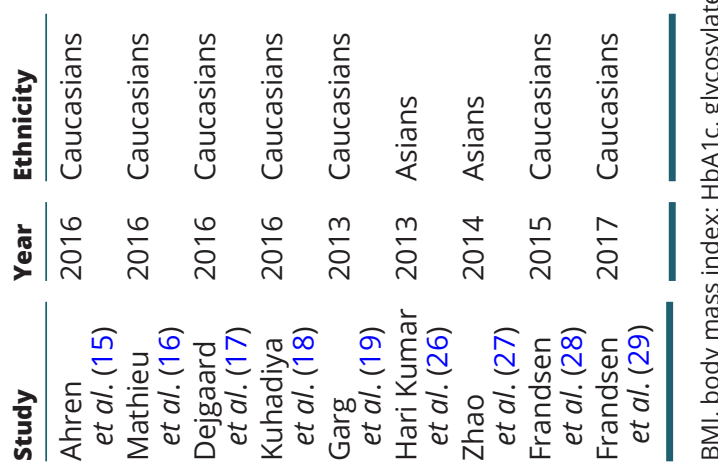

$95 \%$ CI -0.27 to $-0.13, P<0.001)$. However, in the subgroup with a mean baseline BMI $<28 \mathrm{~kg} / \mathrm{m}^{2}$, there was no difference in the reduction in $\mathrm{HbA1c}$ after the incretin-based treatment (WMD 0.00, 95\% CI -0.19 to $0.19, P=0.97$ ) (Table 2).

\section{Secondary outcomes of interest}

\section{Reduction in total daily insulin dose in T1DM patients with incretin-based therapy and subgroup analysis based on incretin-based drug type (GLP-1 RAs or DPP-4 inhibitors)}

Five studies $(17,18,19,26,27)$ reported changes in total daily insulin dose, two of which compared GLP-1 RAs with placebo $(17,18)$. Another two trials compared DPP-4 inhibitors with placebo or insulin alone $(19,27)$, and the remaining trial compared both GLP-1 RA and DPP-4 inhibitor with insulin (26). The meta-analysis showed that incretin-based therapy might reduce the total daily insulin dose with moderate statistical heterogeneity (WMD $-5.53 \mathrm{IU} /$ day, $95 \% \mathrm{CI}-8.89$ to $-2.17, P=0.001$, $\left.I^{2}=51.4 \%\right)$. We performed subgroup analysis based on different types of incretin-based drugs, and the results showed that treatment with GLP-1 RAs significantly reduced the total daily usage of insulin (WMD -9.28 , $95 \%$ CI -15.89 to $\left.-2.68, P=0.006, I^{2}=46.5 \%\right)$. However, treatment with the DPP-4 inhibitor presented no significant difference (WMD $-2.58,95 \% \mathrm{CI}-5.59$ to 0.43 , $P=0.093, I^{2}=0.0 \%$ ) (Fig. 4).

\section{Body weight change in T1DM patients with} incretin-based therapy and subgroup analysis based on mean baseline BMI $\left(\geq 28 \mathrm{~kg} / \mathrm{m}^{2}\right.$ or $\left.<28 \mathrm{~kg} / \mathrm{m}^{2}\right)$, incretin-based drug type (GLP-1 RAs or DPP-4 inhibitors) and liraglutide dose $(1.8,1.2,0.6 \mathrm{mg})$

Eight studies $(15,16,17,18,19,26,28,29)$ reported weight change from baseline to the end of the trial. Figure 5 shows that treatments with incretin-based drugs added to insulin caused a significant reduction in body weight (WMD $-3.24 \mathrm{~kg}, 95 \% \mathrm{CI}-4.43$ to $-2.04, P<0.001$ ), with high heterogeneity among studies $\left(I^{2}=85.4 \%, P<0.001\right)$.

Subgroup analysis showed that there was no significant body weight decrease in the subgroup with a mean baseline $\mathrm{BMI}<28 \mathrm{~kg} / \mathrm{m}^{2}$ (WMD $-2.36,95 \% \mathrm{CI}-5.76$ to $1.03, P=0.17$ ), while body weight decreased remarkably in the other subgroup (BMI $\geq 28 \mathrm{~kg} / \mathrm{m}^{2}$ ) (WMD -3.81 , 95\% CI -4.59 to $-3.03, P<0.001)$ comparison with the placebo control group; therapy with GLP-1 RAs (WMD $-3.86,95 \%$ CI -4.42 to $-3.30, P<0.001)$ but not DPP-4 


\section{B}
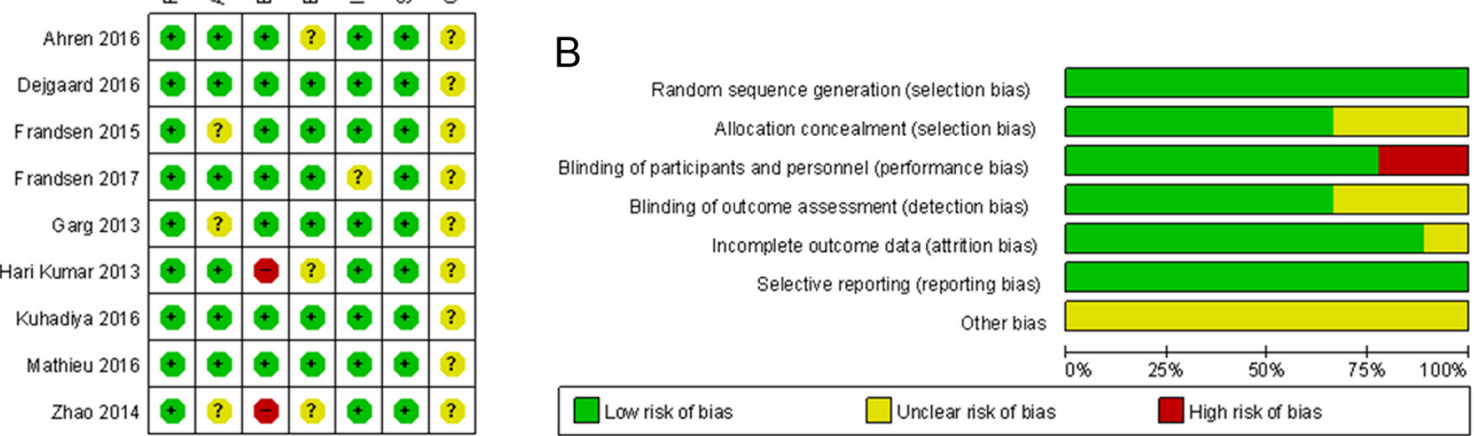

Figure 2

Risk of bias assessment. (A) Summary of risk of bias. (B) Quality assessment percentages in the meta-analysis.

inhibitors (WMD 0.05, 95\% CI -1.11 to $1.21, P=0.93$ ) presented the opposite result (test for subgroup difference: $P=0.41$ ). Body weight was significantly reduced with treatment with $1.8 \mathrm{mg}$ liraglutide (WMD -4.88 , 95\% CI -5.35 to $-4.41, P<0.001$ ), $1.2 \mathrm{mg}$ liraglutide (WMD $-3.82,95 \%$ CI -4.26 to $-3.39, P<0.001)$ and $0.6 \mathrm{mg}$ liraglutide (WMD $-2.21,95 \%$ CI -2.62 to -1.79 , $P<0.001)$. Furthermore, the heterogeneity among groups was significant (test for subgroup difference: $P<0.001$ ) (Table 2).

\section{Hypoglycaemia}

Severe hypoglycaemia was explicitly reported in five studies $(15,16,19,27,28)$. The pooled data did not reveal a higher risk for severe hypoglycaemia upon

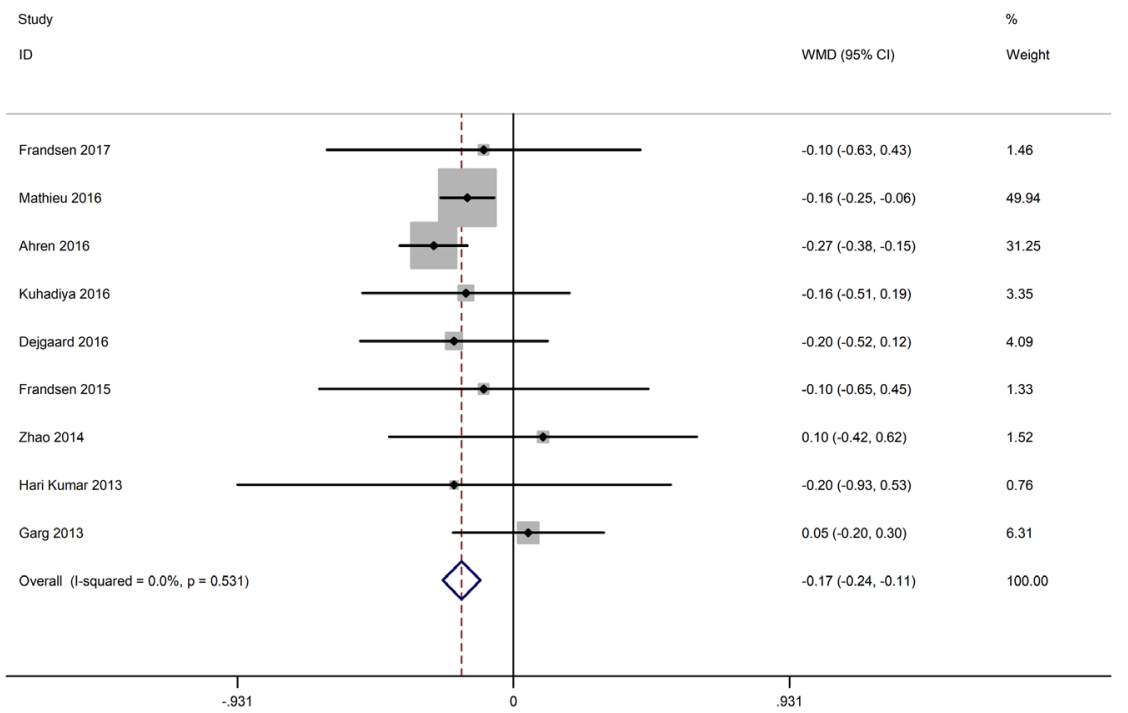

Figure 3

Effects of incretin-based therapies on $\mathrm{HbA} 1 \mathrm{c}$ value. https://ec.bioscientifica.com

https://doi.org/10.1530/EC-18-0546 (c) 2019 The authors Published by Bioscientifica Ltd
This work is licensed under a Creative Commons Attribution-NonCommercial 4.0 International License. ded from Bioscientifica.com at 04/26/2023 07:39:21AM via free access 
Table 2 Subgroup analysis of $\mathrm{HbA} 1 \mathrm{c}$ and body weight.

\begin{tabular}{l}
\hline Outcomes of interest \\
\hline HbA1c (\%) \\
Study design \\
Double-blinded design \\
Open-labelled design \\
Different classes of incretin \\
GLP-1 agonists \\
DPP-4 inhibitors \\
Different doses of liraglutide \\
$1 \mathrm{mg}$ liraglutide \\
$1.2 \mathrm{mg}$ liraglutide \\
$0.6 \mathrm{mg}$ liraglutide \\
Duration of intervention (weeks) \\
$\leq 12$ \\
$>12$ \\
Baseline BMI \\
BMI $<28 \mathrm{~kg} / \mathrm{m}^{2}$ \\
BMI $\geq 28 \mathrm{~kg} / \mathrm{m}^{2}$ \\
Body weight (kg) \\
Mean baseline BMI \\
BMl $<28 \mathrm{~kg} / \mathrm{m}^{2}$ \\
BMI $\geq 28 \mathrm{~kg} / \mathrm{m}^{2}$ \\
Different classes of incretin \\
GLP-1 agonists \\
DPP- 4 inhibitors \\
Different doses of liraglutide \\
$1.8 \mathrm{mg}$ liraglutide \\
$1.2 \mathrm{mg}$ liraglutide \\
$0.6 \mathrm{mg}$ liraglutide \\
\end{tabular}

\begin{tabular}{c}
\hline Studies, no. \\
\hline 9 \\
7 \\
2 \\
7 \\
3 \\
4 \\
5 \\
3 \\
3 \\
6 \\
5 \\
4 \\
8 \\
4 \\
4 \\
7 \\
2 \\
4 \\
\\
4 \\
\hline
\end{tabular}

\begin{tabular}{c}
\hline WMD $(95 \%)$ \\
\hline$-0.17(-0.24,-0.11)$ \\
$-0.18(-0.24,-0.11)$ \\
$0(-0.42,0.42)$ \\
$-0.20(-0.26,-0.13)$ \\
$0.05(-0.17,0.27)$ \\
$-0.24(-0.32,-0.16)$ \\
$-0.20(-0.28,-0.11)$ \\
$-0.15(-0.24,-0.07)$ \\
$-0.13(-0.39,0.13)$ \\
$-0.18(-0.24,-0.11)$ \\
$0.00(-0.19,0.19)$ \\
$-0.20(-0.27,-0.13)$ \\
$-3.24(-4.43,-2.04)$ \\
$-2.36(-5.76,1.03)$ \\
$-3.81(-4.59,-3.03)$ \\
$-3.86(-4.42,-3.30)$ \\
$0.05(-1.11,1.21)$ \\
$-4.88(-5.35,-4.41)$ \\
$-3.82(-4.26,-3.39)$ \\
$-2.21(-2.62,-1.79)$ \\
\hline
\end{tabular}

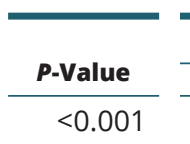

\begin{tabular}{c}
\hline \multicolumn{3}{c}{ Study heterogeneity } \\
\hline$\frac{X^{2}}{7.24} \frac{d f}{7} \frac{I^{2}(\%)}{0} \frac{P \text { value }}{0.51}$
\end{tabular}

1.00

5.96

6

0.67

2.70

0.16

$<0.001$

$<0.001$

2.33

$<0.001$

2.73

0.31

$<0.001$

\subsection{5}

6.89

0.97

$<0.001$

$<0.001$

\subsection{4}

2.4

47.98

0.17

$<0.001$

21.81

$<0.001$

0.93

8.68

$<0.001$

$<0.001$

$<0.001$

9.12
0.11

0.47
1.20
0.22

$8: 3$

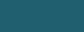

BMI, body mass index; HbA1c, glycosylated haemoglobin; WMD, weighted mean difference.

treatment with incretin-based drugs (OR 0.80, 95\% CI $0.57-1.14, P=0.219)$. The heterogeneity was low $\left(I^{2}=0 \%\right.$, $P=0.577$ ) (Fig. 6).

However, due to inconsistent definitions and reporting of hypoglycaemia, a meta-analysis was not performed for symptomatic hypoglycaemia. Some trials define hypoglycaemia as plasma glucose $(\mathrm{PG})<3.1 \mathrm{mmol} / \mathrm{L}$ or $<3.9 \mathrm{mmol} / \mathrm{L}$ according to different standards. Some reported hours of hypoglycaemia per day, some reported number of patients with hypoglycaemia, and some recorded hypoglycaemia episodes per patient per day.

\section{Gastrointestinal side effects}

Nausea was reported in six of nine studies $(15,16,17,18$, $26,28)$, of which diarrhoea was observed in three studies $(15,17,28)$ and vomiting was observed in four studies $(15,17,18,28)$. As shown in Fig. 7 , in contrast to the control, incretin-based therapy significantly increased the gastrointestinal side effects (OR 3.46, 95\% CI 2.20-5.45, $P<0.001)$ with a high heterogeneity of $62.0 \%$. Subgroup analysis showed that the rate of nausea (OR 4.67,
95\% CI 3.58-6.10, $P<0.001$ ) and vomiting (OR 2.80, 95\% CI $1.15-6.82, P=0.024)$ but not the rate of diarrhoea (OR 1.46, 95\% CI 0.20-10.93, $P=0.710$ ) significantly increased in the incretin-based treated group.

\section{Hyperglycaemia with ketosis}

Two enrolled studies $(15,16)$ reported hyperglycaemia with ketosis, and both studies used liraglutide as an intervention. We pooled the data and performed a metaanalysis. The results (Fig. 8) showed that liraglutide did increase the risk of hyperglycaemia with ketosis (OR 1.62, 95\% CI 1.05-2.49, $I^{2}=46.2 \%, P=0.028$ ). We also performed a subgroup analysis based on the different dosages of liraglutide and found no significant difference in hyperglycaemia with ketosis (test of subgroup difference: $P=0.69$ ) among the group treated with $1.8 \mathrm{mg}$ liraglutide (OR 2.24, 95\% CI 1.03-4.86, $I^{2}=44 \%, P=0.04$ ), the group treated with $1.2 \mathrm{mg}$ liraglutide (OR 1.62, 95\% CI $\left.0.60-4.35, I^{2}=60 \%, P=0.34\right)$ and the group treated with $0.6 \mathrm{mg}$ liraglutide (OR 1.32, 95\% CI 0.50-3.49, $I^{2}=57 \%$, $P=0.57)$. 


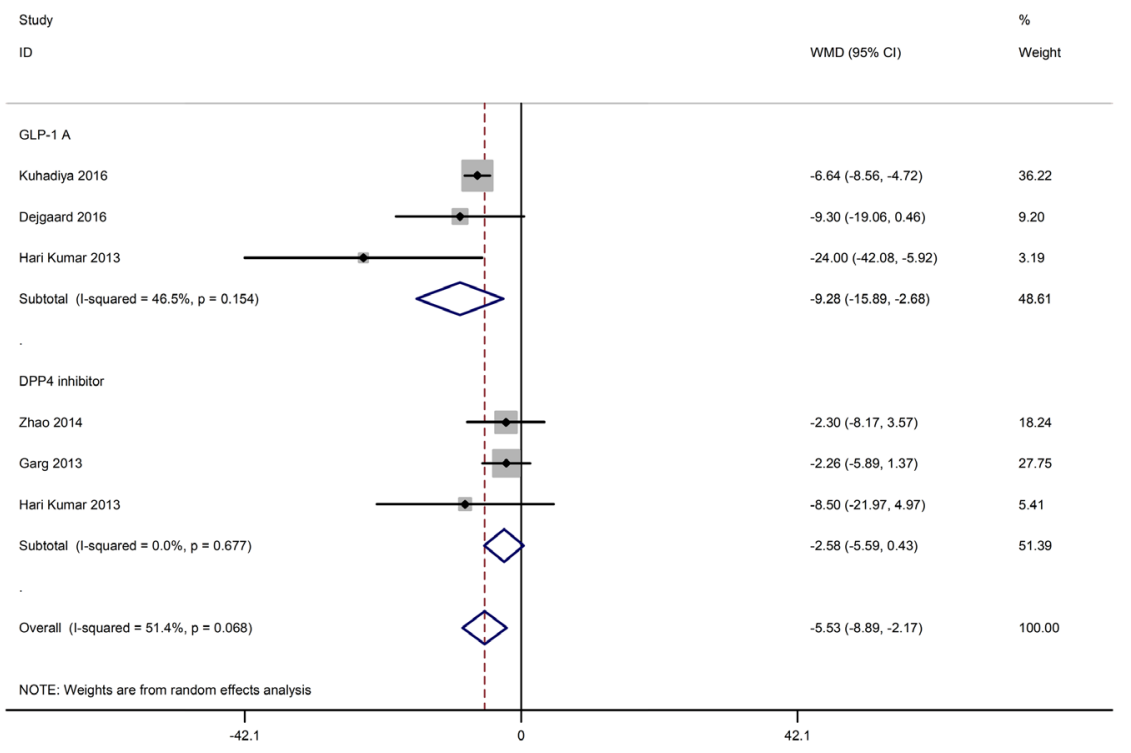

\section{Figure 4}

Effects of incretin-based therapies on total daily insulin dose.

\section{Discussion}

We pooled data from the nine enrolled studies and found that compared to placebo or insulin without added drugs, incretin-based therapy significantly improved glycaemic control, decreased the total daily dose of insulin and reduced body weight without increasing the occurrence of severe hypoglycaemia in T1DM patients. These above data strongly support that incretin-based therapy can improve the management of T1DM.

Subgroup analysis showed that GLP-1 RAs but not DPP-4 inhibitors might reduce HbA1c and that the greater liraglutide dose was, the better the HbA1c-reducing effect. We observed a significant decrease in HbA1c in the subgroup treated for longer than 12 weeks; however, no significant reduction in HbA1c was observed in the subgroup treated with the intervention for a duration less than 12 weeks. It is possible that an intervention duration shorter than 12 weeks is not sufficient to observe an obvious change in $\operatorname{HbA1c}(18,30)$. In the subgroup with a mean baseline BMI $\geq 28 \mathrm{~kg} / \mathrm{m}^{2}$, the body weight and HbA1c significantly decreased after the incretinbased treatment. The effect of lowering body weight could result in improvement in insulin sensitivity (31), thereby contributing to decreased $\mathrm{HbA1c}$ and improved glycaemic control.

The mechanisms by which the gut hormone GLP-1 lowers blood glucose levels are to affect $\beta$ cell (glucosedependent stimulation of insulin production and secretion) and alpha cell (inhibition of glucagon release) function. A paradoxical hyperglucagonaemia in T1DM, which could be attributed to $\beta$ cell destruction and erratic

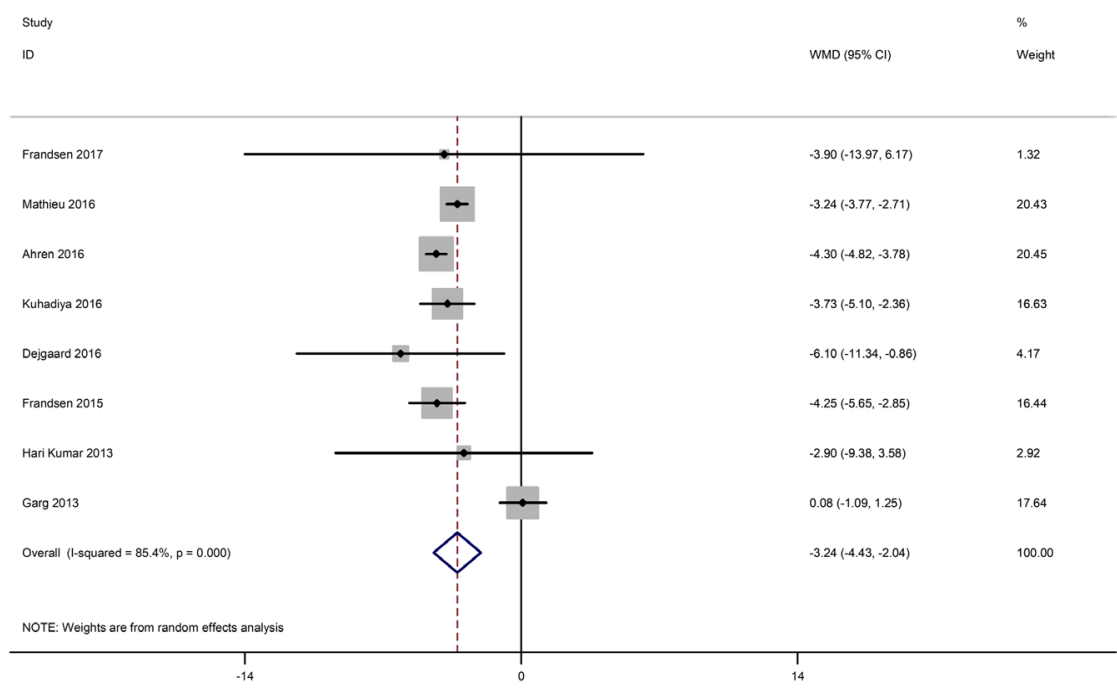

Figure 5

Effects of incretin-based therapies on body weight. https://ec.bioscientifica.com

https://doi.org/10.1530/EC-18-0546 (c) 2019 The authors Published by Bioscientifica Ltd
This work is licensed under a Creative Commons Attribution-NonCommercial 4.0 International License. ded from Bioscientifica.com at 04/26/2023 07:39:21AM 


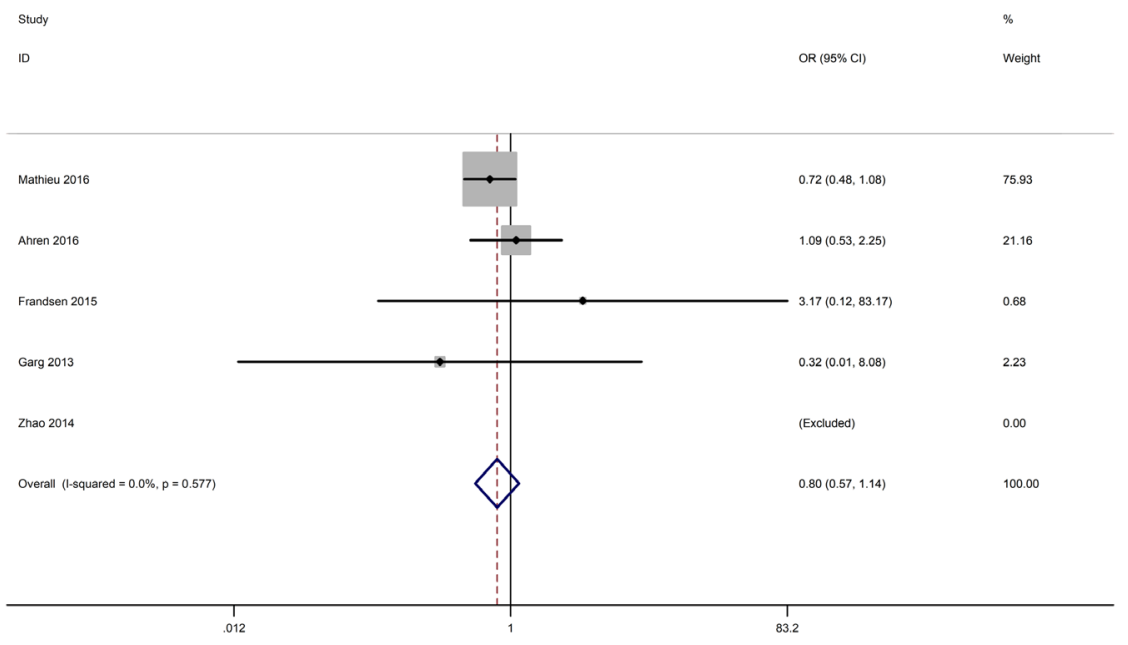

\section{Figure 6}

Effects of incretin-based therapies on the risk of severe hypoglycaemia. blood glucose control, results in uncontrolled hepatic glucose production (32); thus, GLP-1 agonists could be used for T1DM. Additionally, GLP-1 has extrapancreatic effects, including a reduction in gastric emptying and intestinal glucose uptake, promotion of satiety and suppression of hepatic glucose production, resulting in reduced food intake and weight loss (8). Recently, increasing evidence has demonstrated the beneficial effects of GLP-1 on $\beta$ cell function because GLP- 1 has been shown to improve $\beta$ cell function and survival in vitro and in several rodent models of diabetes (33). Therefore, incretin-based treatment offers possibilities that would benefit patients with T1DM.

Although our results strengthen the evidence supporting the efficacy of incretins, we did not pool the data about hypoglycaemia due to different definitions and the diversity of methods applied to assess outcomes. However, we performed a meta-analysis of the occurrence of severe hypoglycaemia, and the results indicated that incretins did not contribute to severe hypoglycaemia. This may partly be due to that liraglutide does not impair glucagon counter-regulation of hypoglycaemia (34) and DPP-4 inhibitors did not cause severe hypoglycaemia in T1DM (35).

Additionally, we found that incretin-based treatment did have a relationship with the risk of hyperglycaemia with ketosis. And the subgroup analysis based on liraglutide dosage showed that hyperglycaemia with ketosis may increase moderately in the group treated with

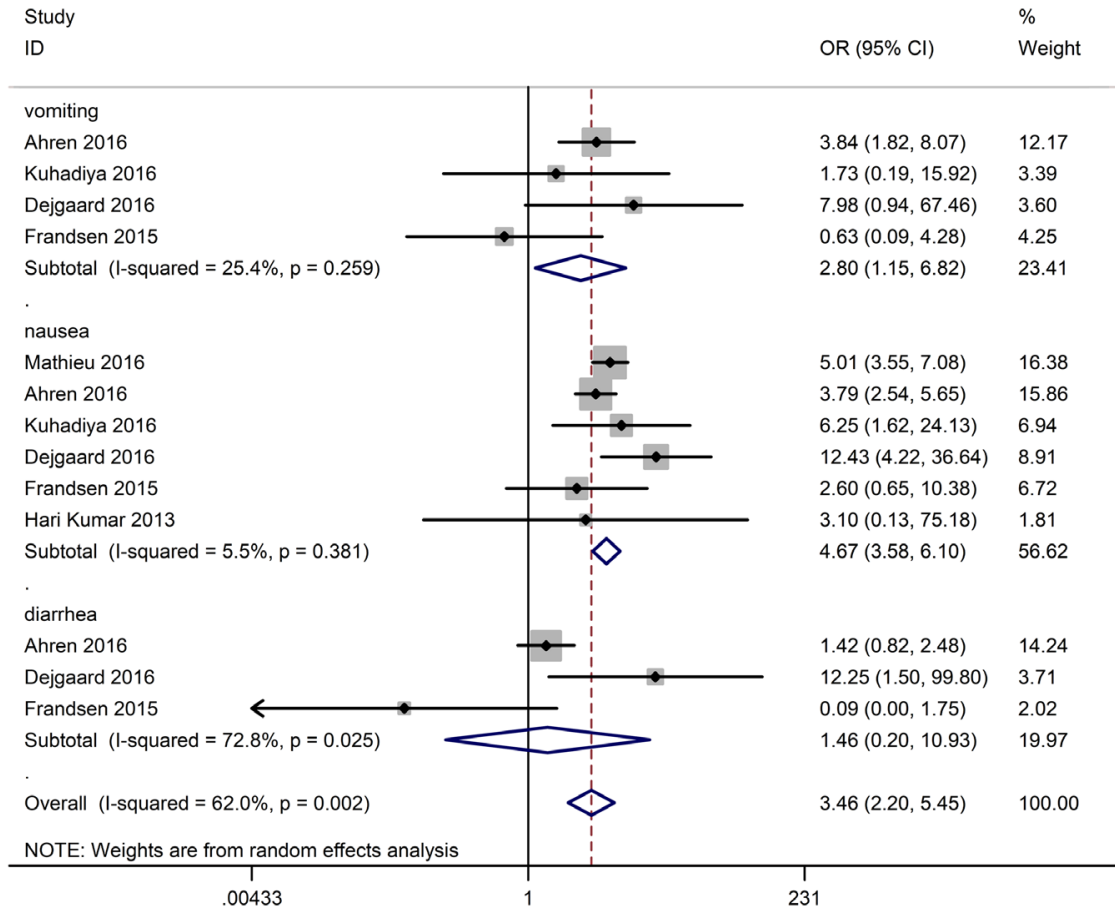

Figure 7

Effects of incretin-based therapies on gastrointestinal side effects. https://ec.bioscientifica.com

https://doi.org/10.1530/EC-18-0546 (c) 2019 The authors Published by Bioscientifica Ltd
This work is licensed under a Creative Commons Attribution-NonCommercial 4.0 International License. ded from Bioscientifica.com at 04/26/2023 07:39:21AM 
$1.8 \mathrm{mg}$ liraglutide. This finding could be explained by the reduced insulin dose in the group with the large dose of liraglutide, which may lead to ketone production (16). Moreover, due to just two small studies included and the great heterogeneity among groups, the results should be interpreted with caution.

Furthermore, our study also found that GLP-1 RAs increased the risk of gastrointestinal side effects, such as vomiting and nausea, but not diarrhoea. Among the nine enrolled trials included in our meta-analysis, six trials $(15,16,17,18,26,28)$ reported adverse gastrointestinal effects, including nausea, diarrhoea and vomiting. Five trials $(15,16,17,18,28)$ only used GLP-1 RAs for treatment, and the remaining trial (26) used either GLP-1 RA or a DPP-4 inhibitor. However, the latter only reported gastrointestinal disorders related to GLP-1 RA but not the DPP-4 inhibitor. Thus, the gastrointestinal side effects were all related to GLP-1 RAs, and there were no reports regarding the gastrointestinal adverse effects of DPP-4 inhibitors in T1DM. Therefore, further studies investigating DPP-4 inhibitors are warranted to explore the gastrointestinal adverse events in patients with T1D. In addition, the same gastrointestinal side effects were observed in the patients with type 2 diabetes who were treated with GLP-1 RAs (36).

To the best of our knowledge, this is the most accurate and comprehensive meta-analysis of incretin-based therapy without other classified antidiabetic drugs in T1DM. In 2016, Guo et al. (35) conducted a meta-analysis of six RCTs investigating the efficacy and safety of DPP-4 inhibitors in T1DM. The authors concluded that DPP-4 inhibitors could not show any advantage in decreasing HbA1c levels in patients with T1DM. In 2016, Wang et al. (37) performed a meta-analysis of 12 studies to clarify the efficacy and safety of incretin-based drugs in patients with T1DM. They found that treatment of incretin-based drugs in patients with T1DM was significantly associated with reduced HbA1c and weight loss. However, the authors pooled analyses, including combination therapy and active drug-controlled and placebo-controlled studies. We provided an updated overview, and our analysis excluded clinical trials using an active drug as a comparator.

There are several strengths of our meta-analysis. Most importantly, we used multiple strategies and extensive literature searches to identify studies and adopted rigorous criteria for including studies. Moreover, subgroup analysis was conducted according to the Cochrane handbook to minimize the heterogeneity. Furthermore, a recent trial was incorporated to better clarify the effects of incretinbased therapy on HbA1c and body weight in T1DM patients (16). Moreover, the studies included in our meta-analysis were all RCTs with high quality. Finally, we searched ClinicalTrials.gov for more detailed information to ensure that the data were accurate.

However, the following limitations of our metaanalysis must be considered. First, a very large variation existed in the sample sizes of the included studies, which ranged from 17 to 1389 cases. Significant differences were also noted regarding study design, type of incretinbased drug and dose of the GLP-1 RA liraglutide. Second, the results might be influenced by two of the included open-label studies because these studies were not blinded.

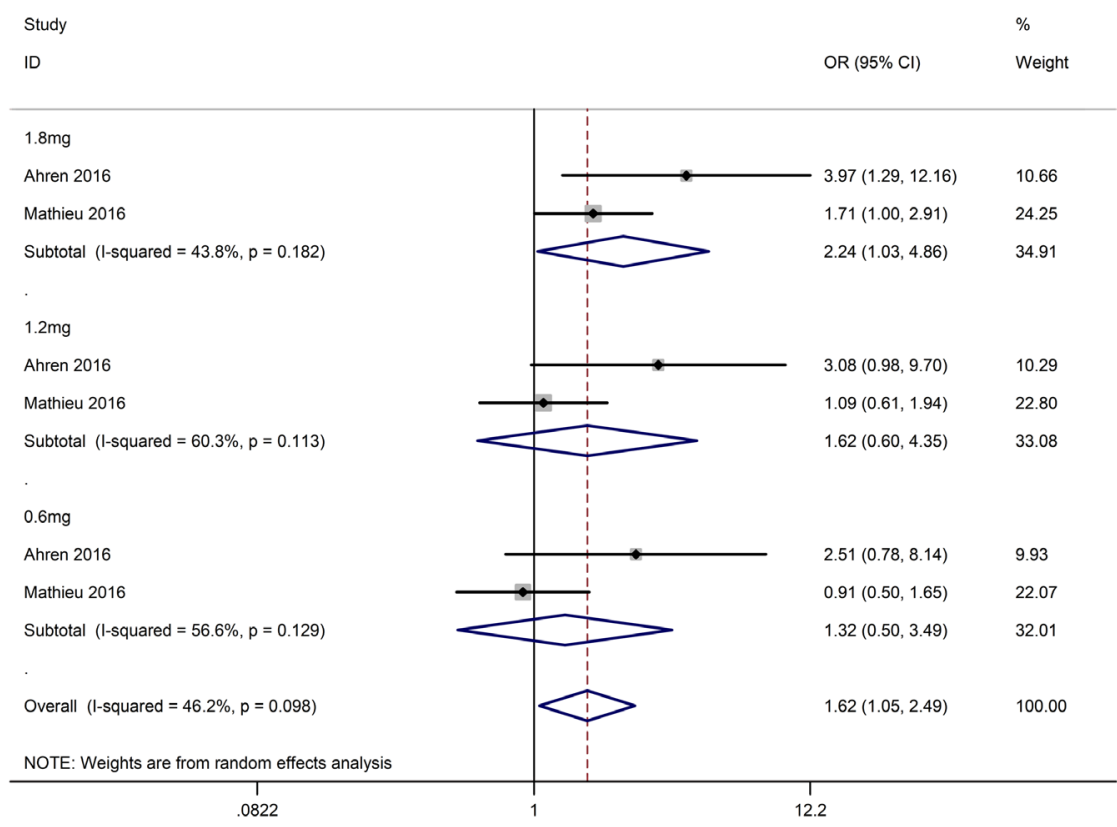

\section{Figure 8}

Effects of incretin-based therapies on hyperglycaemia with ketosis. 
Third, we did not include observational studies. Fourth, we excluded some studies because of the unavailability of original data, which could contribute to inaccuracy of the risk estimates. Finally, we did not include unpublished grey studies, which mainly provided negative results. The drawbacks mentioned above may lead to moderate heterogeneity. The results based on heterogeneous data should be interpreted carefully although subgroup analysis was applied.

\section{Conclusions}

In summary, the available evidence indicated that GLP-1 RAs, but not DPP4 inhibitors, along with insulin in patients with T1DM increase the efficacy of glycaemic control without increasing the rate of severe hypoglycaemia. The adverse events associated with incretin-based treatments were moderate. Further studies with greater power are warranted to explore the side effects of incretins.

\section{Supplementary data}

This is linked to the online version of the paper at https://doi.org/10.1530/ EC-18-0546.

\section{Declaration of interest}

The authors declare that there is no conflict of interest that could be perceived as prejudicing the impartiality of the research reported.

\section{Funding}

This work was supported by a National Natural Science Foundation of China (NSFC)-7 Research Grants Council (RGC) grant (81461168031, to Z Z), NSFC grants ( 81670772,81870577 , to Y X and 81774112 , to Z M).

\section{Author contribution statement}

Zhou $Z$, Xiao $Y$ and Liu $L$ designed the study. Xiao $Y$ searched the library; Liu $L$ reviewed and selected studies. Liu $L$ wrote the manuscript, and Shao $Z$ assisted in manuscript revision. Xia Y, Qin J and Mei Z analysed and interpreted the data. Zhou $Z$ and Xiao $Y$ took full responsibility for the integrity and accuracy of the data. All authors approved the manuscript.

\section{References}

1 Burn P. Type 1 diabetes. Nature Reviews Drug Discovery 20109 187-188. (https://doi.org/10.1038/nrd3097)

2 American Diabetes Association. 8. Pharmacologic approaches to glycemic treatment: standards of medical care in diabetes-2018. Diabetes Care 201841 (Supplement 1) S73-S85. (https://doi. org/10.2337/dc18-S008)

3 Diabetes Control and Complications Trial Research Group, Nathan DM, Genuth S, Lachin J, Cleary P, Crofford O, Davis M, Rand L \& Siebert C. The effect of intensive treatment of diabetes on the development and progression of long-term complications in insulin-dependent diabetes mellitus. New England Journal of Medicine 1993329 977-986. (https://doi.org/10.1056/ NEJM199309303291401)

4 Diabetes Control and Complications Trial Research Group. Adverse events and their association with treatment regimens in the diabetes control and complications trial. Diabetes Care 199518 1415-1427. (https://doi.org/10.2337/diacare.18.11.1415)

5 Holst JJ. Incretin hormones and the satiation signal. International Journal of Obesity 201337 1161-1168. (https://doi.org/10.1038/ ijo.2012.208)

6 Herman GA, Stein PP, Thornberry NA \& Wagner JA. Dipeptidyl peptidase- 4 inhibitors for the treatment of type 2 diabetes: focus on sitagliptin. Clinical Pharmacology and Therapeutics 200781 761-767. (https://doi.org/10.1038/sj.clpt.6100167)

7 Gutniak M, Orskov C, Holst JJ, Ahren B \& Efendic S. Antidiabetogenic effect of glucagon-like peptide-1 (7-36) amide in normal subjects and patients with diabetes mellitus. New England Journal of Medicine 1992326 1316-1322. (https://doi.org/10.1056/ NEJM199205143262003)

8 Campbell JE \& Drucker DJ. Pharmacology, physiology, and mechanisms of incretin hormone action. Cell Metabolism 201317 819-837. (https://doi.org/10.1016/j.cmet.2013.04.008)

9 Jonas D, Van Scoyoc E, Gerrald K, Wines R, Amick H, Triplette M \& Runge T. Drug Class Review: Newer Diabetes Medications, TZDs, and Combinations: Final Original Report. Portland, OR, USA: Oregon Health \& Science University, 2011. (https://doi.org/10.1002/0470846399.ch23)

10 Zhao Y, Yang L \& Zhou Z. Dipeptidyl peptidase-4 inhibitors: multitarget drugs, not only antidiabetes drugs. Journal of Diabetes 20146 21-29. (https://doi.org/10.1111/1753-0407.12063)

11 Zhao Y, Yang L, Wang X \& Zhou Z. The new insights from DPP-4 inhibitors: their potential immune modulatory function in autoimmune diabetes. Diabetes/Metabolism Research and Reviews 2014 30 646-653. (https://doi.org/10.1002/dmrr.2530)

12 Eng C, Kramer CK, Zinman B \& Retnakaran R. Glucagon-like peptide-1 receptor agonist and basal insulin combination treatment for the management of type 2 diabetes: a systematic review and meta-analysis. Lancet 2014384 2228-2234. (https://doi.org/10.1016/ S0140-6736(14)61335-0)

13 Cao CQ, Xiang YF \& Zhou ZG. The clinical application of linagliptin in Asians. Therapeutics and Clinical Risk Management 201511 1409-1419. (https://doi.org/10.2147/TCRM.S64402)

14 Lovshin JA \& Drucker DJ. Incretin-based therapies for type 2 diabetes mellitus. Nature Reviews Endocrinology 20095 262-269. (https://doi. org/10.1038/nrendo.2009.48)

15 Ahren B, Hirsch IB, Pieber TR, Mathieu C, Gomez-Peralta F, Hansen TK, Philotheou A, Birch S, Christiansen E, Jensen TJ, et al. Efficacy and safety of liraglutide added to capped insulin treatment in subjects with type 1 diabetes: the ADJUNCT TWO randomized trial. Diabetes Care 201639 1693-1701. (https://doi.org/10.2337/ dc16-0690)

16 Mathieu C, Zinman B, Hemmingsson JU, Woo V, Colman P, Christiansen E, Linder M, Bode B \& ADJUNCT ONE Investigators. Efficacy and safety of liraglutide added to insulin treatment in type 1 diabetes: the ADJUNCT ONE treat-to-target randomized trial. Diabetes Care 201639 1702-1710. (https://doi.org/10.2337/dc160691)

17 Dejgaard TF, Frandsen CS, Hansen TS, Almdal T, Urhammer S, Pedersen-Bjergaard U, Jensen T, Jensen AK, Holst JJ, Tarnow L, et al. Efficacy and safety of liraglutide for overweight adult patients with type 1 diabetes and insufficient glycaemic control (Lira-1): a randomised, double-blind, placebo-controlled trial. Lancet Diabetes and Endocrinology 20164 221-232. (https://doi.org/10.1016/S22138587(15)00436-2)

18 Kuhadiya ND, Dhindsa S, Ghanim H, Mehta A, Makdissi A, Batra M, Sandhu S, Hejna J, Green K, Bellini N, et al. Addition of liraglutide

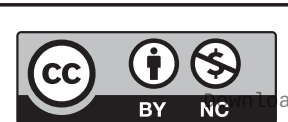

This work is licensed under a Creative Commons Attribution-NonCommercial 4.0 International License. ded from Bioscientifica com at 04/26/2023 07:39:21AM 
to insulin in patients With type 1 diabetes: a randomized placebocontrolled clinical trial of 12 weeks. Diabetes Care 201639 1027-1035. (https://doi.org/10.2337/dc15-1136)

19 Garg SK, Moser EG, Bode BW, Klaff LJ, Hiatt WR, Beatson C \& SnellBergeon JK. Effect of sitagliptin on post-prandial glucagon and GLP-1 levels in patients with type 1 diabetes: investigator-initiated, doubleblind, randomized, placebo-controlled trial. Endocrine Practice 2013 19 19-28. (https://doi.org/10.4158/EP12100.OR)

20 Shamseer L, Moher D, Clarke M, Ghersi D, Liberati A, Petticrew M, Shekelle P, Stewart LA \& PRISMA-P Group. Preferred reporting items for systematic review and meta-analysis protocols (PRISMA-P) 2015: elaboration and explanation. BMJ $2015350 \mathrm{~g} 7647$. (https://doi. org/10.1136/bmj.g7647)

21 Zhou Z, Xiang Y, Ji L, Jia W, Ning G, Huang G, Yang L, Lin J, Liu Z, Hagopian WA, et al. Frequency, immunogenetics, and clinical characteristics of latent autoimmune diabetes in China (LADA China Study) a nationwide, multicenter, clinic-based cross-sectional study. Diabetes 201362 543-550. (https://doi.org/10.2337/db12-0207)

22 Wan X, Wang W, Liu J \& Tong T. Estimating the sample mean and standard deviation from the sample size, median, range and/or interquartile range. BMC Medical Research Methodology 201414135. (https://doi.org/10.1186/1471-2288-14-135)

23 Higgins JPT, Altman DG, Gotzsche PC, Juni P, Moher D, Oxman AD, Savovic J, Schulz KF, Weeks L \& Sterne JAC. The Cochrane Collaboration's tool for assessing risk of bias in randomised trials. BMJ 2011343 d5928. (https://doi.org/10.1136/bmj.d5928)

24 Egger M, Davey Smith G, Schneider M \& Minder C. Bias in metaanalysis detected by a simple, graphical test. BMJ 1997315 629-634. (https://doi.org/10.1136/bmj.315.7109.629)

25 Higgins JP, Thompson SG, Deeks JJ \& Altman DG. Measuring inconsistency in meta-analyses. BMJ 2003327 557-560. (https://doi. org/10.1136/bmj.327.7414.557)

26 Hari Kumar KV, Shaikh A \& Prusty P. Addition of exenatide or sitagliptin to insulin in new onset type 1 diabetes: a randomized, open label study. Diabetes Research and Clinical Practice 2013100 e55-e58. (https://doi.org/10.1016/j.diabres.2013.01.020)

27 Zhao Y, Yang L, Xiang Y, Liu L, Huang G, Long Z, Li X, Leslie RD, Wang X \& Zhou Z. Dipeptidyl peptidase 4 inhibitor sitagliptin maintains beta-cell function in patients with recent-onset latent autoimmune diabetes in adults: one year prospective study. Journal of Clinical Endocrinology and Metabolism 201499 E876-E880. (https:// doi.org/10.1210/jc.2013-3633)

28 Frandsen CS, Dejgaard TF, Holst JJ, Andersen HU, Thorsteinsson B $\&$ Madsbad S. Twelve-week treatment with liraglutide as add-on to insulin in normal-weight patients with poorly controlled type 1 diabetes: a randomized, placebo-controlled, double-blind parallel study. Diabetes Care 201538 2250-2257. (https://doi.org/10.2337/ dc15-1037)

29 Frandsen CS, Dejgaard TF, Andersen HU, Holst JJ, Hartmann B, Thorsteinsson B \& Madsbad S. Liraglutide as adjunct to insulin treatment in type 1 diabetes does not interfere with glycaemic recovery or gastric emptying rate during hypoglycaemia: a randomized, placebo-controlled, double-blind, parallel-group study. Diabetes, Obesity and Metabolism 201719 773-782. (https://doi. org/10.1111/dom.12830)

30 Desouza CV, Rosenstock J, Zhou R, Holcomb RG \& Fonseca VA. Glycated albumin at 4 weeks correlates WITH A1C levels at 12 weeks and reflects short-term glucose fluctuations. Endocrine Practice 2015 21 1195-1203. (https://doi.org/10.4158/EP14570.OR)

31 Vink RG, Roumans NJ, Cajlakovic M, Cleutjens JPM, Boekschoten MV, Fazelzadeh P, Vogel MAA, Blaak EE, Mariman EC, van Baak MA, et al. Diet-induced weight loss decreases adipose tissue oxygen tension with parallel changes in adipose tissue phenotype and insulin sensitivity in overweight humans. International Journal of Obesity 201741 722-728. (https://doi.org/10.1038/ijo.2017.38)

32 Lee Y, Wang MY, Du XQ, Charron MJ \& Unger RH. Glucagon receptor knockout prevents insulin-deficient type 1 diabetes in mice. Diabetes 201160 391-397. (https://doi.org/10.2337/db10-0426)

33 Zummo FP, Cullen KS, Honkanen-Scott M, Shaw JAM, Lovat PE $\&$ Arden C. Glucagon-like peptide 1 protects pancreatic beta-cells from death by increasing autophagic flux and restoring lysosomal function. Diabetes 201766 1272-1285. (https://doi.org/10.2337/ db16-1009)

34 Pieber TR, Deller S, Korsatko S, Jensen L, Christiansen E, Madsen J \& Heller SR. Counter-regulatory hormone responses to hypoglycaemia in people with type 1 diabetes after 4 weeks of treatment with liraglutide adjunct to insulin: a randomized, placebo-controlled, double-blind, crossover trial. Diabetes, Obesity and Metabolism 2015 17 742-750. (https://doi.org/10.1111/dom.12473)

35 Guo H, Fang C, Huang Y, Pei Y, Chen L \& Hu J. The efficacy and safety of DPP4 inhibitors in patients with type 1 diabetes: a systematic review and meta-analysis. Diabetes Research and Clinical Practice 2016121 184-191. (https://doi.org/10.1016/j. diabres.2016.08.022)

36 Amori RE, Lau J \& Pittas AG. Efficacy and safety of incretin therapy in type 2 diabetes: systematic review and meta-analysis. JAMA 2007 298 194-206. (https://doi.org/10.1001/jama.298.2.194)

37 Wang W, Gao Y, Chen D, Wang C, Feng X \& Ran X. Efficacy and safety of incretin-based drugs in patients with type 1 diabetes mellitus: a systematic review and meta-analysis. Diabetes Research and Clinical Practice 2017129 213-223. (https://doi.org/10.1016/j. diabres.2017.05.007)

Received in final form 8 January 2019

Accepted 28 January 2019

Accepted Preprint published online 29 January 2019 https://ec.bioscientifica.com https://doi.org/10.1530/EC-18-0546 (c) 2019 The authors Published by Bioscientifica Ltd

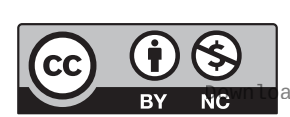

This work is licensed under a Creative Commons Attribution-NonCommercial 4.0 International License. ded from Bioscientifica.com at 04/26/2023 07:39:21AM 